\title{
Vitamin and mineral supplementation effect on muscular activity and cycling efficiency in master athletes
}

\author{
Julien Louis, Christophe Hausswirth, François Bieuzen, and Jeanick Brisswalter \\ J. Louis and J. Brisswalter. Laboratory of Human Motricity, Education, and Health, \\ University of Nice Sophia Antipolis, BP 3259, 06205 Nice, France. (e-mail: \\ brisswalter@gmail.com). \\ C. Hausswirth and F. Bieuzen. Laboratoire de Biomécanique et de Physiologie, Institut \\ National du Sport et de L’Education Physique (INSEP), Paris 75012, France.
}

Publié dans : Applied physiology, nutrition and metabolism (ISSN 1715-5312), 2010, vol. 35, n³, pp. 251-260 DOI : 10.1139/H10-014 PMID : 20555368

\begin{abstract}
The influence of vitamin and mineral complex supplementation on muscular activity and cycling efficiency was examined in elderly endurance-trained master athletes during a heavy cycling trial. Master athletes were randomly assigned in a double-blind process to 1 of 2 treatment groups: antioxidant supplementation ( $n=8$ : As group) or placebo ( $n=8$ : Pl group) for 21 days. After that time, each subject had to perform a 10-min session of cycling on a cycloergometer at a heavy constant intensity. Twenty-four to $48 \mathrm{~h}$ after this session, subjects performed an isometric maximal voluntary contraction before and immediately after a fatiguing strength training (leg press exercise) and the same 10-min cycling test after fatigue. Isometric maximal voluntary force (MVF) of knee extensors was assessed before and after fatigue. Electromyographic (EMG) activity of the vastus medialis, the vastus lateralis (VL), and the biceps femoris was recorded with surface EMG. The knee-extensors MVF after the fatiguing exercise was reduced in similar proportions for both groups (As, $-10.9 \%$; $\mathrm{Pl},-11.3 \%, p<0.05$ ). This MVF loss was associated with a significant reduction in EMG frequency parameters for both groups, with a lower decrease for the As group. Muscular activity and cycling efficiency during the cycling bouts were affected by the treatment. Cycling efficiency decreased significantly and the oxygen uptake slow component was higher after the fatiguing exercise for both groups. Furthermore, a decrease in cycling efficiency was associated with an increase in VL activity. However, these changes were significantly lower for the As group. The results of the present study indicate an overall positive effect of vitamin and mineral complex supplementation on cycling efficiency after fatigue, in the endurance-trained elderly.

Key words: aging, electromyography, cycling efficiency, maximal voluntary contraction, master
\end{abstract} athlete.

Résumé : L’objet de cette étude était d'analyser l'influence d'une supplémentation en vitamines et minéraux sur la performance musculaire et le rendement au cours d'un exercice de cyclisme intense. Deux groupes de 8 personnes âgées régulièrement entrainées en endurance, ont été constitués selon une procédure en double aveugle avec l'apport soit d'un complexe d'antioxydants ( $n=8$ : groupe As), soit d'un placebo ( $n=8:$ groupe $\mathrm{Pl}$ ) pendant 21 jours. Après 21 jours de supplémentation chaque sujet devait effectuer une session de cyclisme de 10 min à 
une intensité intense. 24 à 48 h après cette session les sujets réalisait un test d'évaluation de la force maximale volontaire (FMV) avant et après une séance d'entrainement en résistance et le même exercice de cyclisme après fatigue. La force maximale isométrique volontaire (FMV) des extenseurs du genou était testée avant et après fatigue. L'activité électromyographique (EMG) du vastus medialis (VM), vastus lateralis (VL) et biceps femoris (BF) était enregistrée à partir d'électrodes de surface. La FMV des muscles extenseurs du genou était réduite après l'exercice fatigant, dans des proportions similaires pour les 2 groupes (As, $-10,9 \%$; $\mathrm{Pl},-11,3 \%, p<0,05$ ). Cette baisse de la FMV était accompagnée d'une réduction des paramètres EMG fréquentiels, pour les 2 groupes, avec toutefois une moindre diminution pour le groupe As. La tolérance à l'exercice en cyclisme était modifiée entre les 2 sessions. Le rendement en cyclisme était réduit et la composante lente de consommation d'oxygène était augmentée pour les 2 groupes après l'exercice fatigant. Cette altération du rendement en cyclisme était accompagnée d'une augmentation de l'activité musculaire du VL. Néanmoins ces changements étaient significativement moindres pour le groupe As. Les résultats de cette étude suggèrent un effet bénéfique d'une supplémentation en vitamines et minéraux sur le rendement en cyclisme après fatigue, chez des personnes âgées entraînées en endurance.

Mots-clés : vieillissement, électromyographie, rendement en cyclisme, force maximale volontaire, athlète master.

\section{Introduction}

In the recent years, the number of elderly people (i.e., >60 years) participating in competitive events has increased (Lepers 2008; Tanaka and Seals 2008). These athletes, commonly called “'master athletes", are healthier than age-matched people in virtually every category that has been measured. For example, some master athletes older than 70 years are able to run a marathon in $3 \mathrm{~h}$ (Tanaka and Seals 2008). Within this framework, there has been increased interest in issues related to the maintenance or even enhancement of the performance of master athletes (Lepers 2008; Tanaka and Seals 2008). The main findings of these studies indicate that master athletes are able to maintain their performance level despite the structural and functional physical capacity changes classically described with aging (Lexell 1995; Tanaka and Seals 2008). Determination of the effects of aging on these changes is difficult because of the confounding effects of reductions in physical activity, changes in body composition, and development of clinical diseases. Therefore, the study of highly trained subjects could represent an alternative approach to analyzing the effects of aging on physical performance.

Most studies reporting on the effect of age on performance have shown an impairment of maximal force generating capacities and a reduction in maximal aerobic capacity, leading to a lower endurance capacity in elderly compared with young adults (Lepers 2008; Tanaka and Seals 2008). Therefore, in recent studies, much attention has been given to the need to identify factors affecting performance decrease and to strategies to increase performance in the older population (Deley et al. 2001; Louis et al. 2009). Among factors affecting the force-generating decline and the endurance reduction associated with aging, the role of free-radical damages in muscular function is classically evoked (Warren et al. 1992; Figueiredo et al. 2008). Furthermore, in endurance-trained subjects, prolonged exercise with a high level of oxygen consumption ( VO2) is associated with oxygen radicals production, exceeding the cellular antioxidant system and resulting in the peroxydation of polyunsatured fatty acids in membrane structures (Mastaloudis et al. 2004). Within this framework, antioxidant supplementation might attenuate the loss of muscle function and could be a complementary strategy for reducing the performance alteration 
associated with aging, especially in endurance-trained subjects. A complex of vitamins and minerals is frequently used by athletes during training and competition periods.

These complexes usually contain some substances recognized for their antioxidant effects, such as vitamin $\mathrm{C}$, vitamin E, b-carotene, zinc, selenium, and manganese. Because antioxidants work together to protect organisms from the potential side effects of free radicals (Sen and Hanninen 1994), previous studies have attempted to investigate the effectiveness on performance of a combination of several antioxidants as supplements (Mastaloudis et al. 2004; Goldfarb et al. 2005; Gauche' et al. 2006). The main findings indicate a protective effect on muscular function of such supplementation, principally by controlling the toxic effects of free radicals and preventing lipid peroxydation. Recently, Gauche' et al. (2006) reported a faster muscular force recovery after a prolonged trail running race in young, endurance-trained subjects. Therefore, antioxidant supplementation could provide beneficial effects on muscle performance. Nevertheless, little information is available about the effectiveness of antioxidant supplementation in preventing exercise-related muscle alterations in elderly endurance-trained subjects.

Muscular performance alterations can be analyzed with isometric or dynamic maximal voluntary contraction (MVC) performed before and after a fatiguing protocol, by analyzing changes in muscular activity (Hausswirth et al. 2000; Lattier et al. 2004). On the other hand, during locomotion, it has been well documented that gross efficiency can be considered an important determinant of exercise tolerance (di Prampero 1986; Hausswirth and Brisswalter 2008). Furthermore, during a heavy constant-load exercise above lactate threshold, the attainment of a metabolic steady state may not occur and a secondary component of increasing oxygen uptake ( VO2) slow component (SC) may be observed that reflects mainly muscular activity and the appearance of fatigue phenomenon (Poole et al. 1994; Vercruyssen et al. 2008).

In this context, the aim of the present study was to examine the effect of vitamin and mineral supplementation on muscular activity and exercise tolerance in elderly people regularly trained in endurance. We postulated that a 21-day period of vitamin and mineral supplementation prior to a fatiguing exercise may improve muscular function and exercise tolerance in elderly endurancetrained subjects.

\section{Materials and methods}

\section{Approach to the problem and experimental design}

This experiment was conducted to examine the effect of vitamin and mineral supplementation on muscular activity and cycling efficiency during a heavy constant-load cycling exercise performed before and after a fatiguing strength exercise on a leg press. This strength exercise was used as a model to create fatigue, and allowed us to study adaptative mechanisms to fatigue (Ferri et al. 2006; Louis et al. 2009). The experimental protocol was composed of 3 sessions of tests in the laboratory. The first session was performed 1 month before the experiment. The 2 other sessions were performed in a 1-week period, during which subjects were asked not to train between sessions. Dependent variables included isometric maximal voluntary force (MVF) of knee extensor muscles and corresponding electromyographic (EMG) activity, gross efficiency, VO2SC, and EMG activity recorded during the heavy cycling exercise. Measurements were performed before and after the fatiguing exercise. Dependent variables were analyzed as a function of independent variables (i.e., time and supplementation) (Fig. 1). 


\section{Subjects}

Sixteen elderly (>60 years) endurance-trained athletes (age, $66.1 \pm 5.8$ years; height, $1.75 \pm 0.06$ $\mathrm{m}$; body mass, $76.4 \pm 7.1 \mathrm{~kg}$ ) volunteered to participate in this study. All participants were informed of the study procedures and risks before participation. Subjects had to be free from present or past neuromuscular and metabolic pathology that could affect measured variables. No subjects had used nutritional supplementation for the 6 weeks before the experimental protocol. Particular care was taken in recruiting individuals with similar activity levels and body stature. The individuals selected were regularly trained subjects engaged in cycling and running long distances at least 2 times per week (mean training time per week: 6 h cycling and $1 \mathrm{~h}$ running). Written informed consent was obtained from the participants, and the study was conducted according to the Declaration of Helsinki. Before its initiation, this study was approved by the local ethics committee (St. Germain en Laye, France).

\section{Experimental protocol}

\section{Preliminary session: determination of dominant leg and VO2 max, and randomization to treatment group}

During an initial session that took place 1 month before the experiment, each subject performed 2 tests. The first test was aimed at determining their dominant leg by kicking a ball according to the method described by Daly and Cavanagh (1976). The second test was an incremental cycling test performed at a self-selected cadence on a cycle ergometer (SRM, Schoberer Rad Messtecnik, Ju"lich, Welldorf, Germany). The handlebars and racing seat of the ergocycle were fully adjustable both vertically and horizontally to reproduce positions used by subjects on their own bicycles. Moreover, this ergometer was equipped with individual racing pedals and toes clips, allowing subjects to wear their own shoes. This ergometer allowed subjects to maintain a constant power output independent of the selected cadence, by automatically adjusting torque to angular velocity. The test was performed according to the recommendations of the French Sport Medicine Society. The test began with a 6-min warm-up at $100 \mathrm{~W}$, after which the power output was increased by $30 \mathrm{~W}$ every 1 min until voluntary exhaustion. During this incremental exercise, _VO2, minute ventilation, and respiratory exchange ratio (RER) were recorded continuously using a cycle-to-cycle metabolic portable system (Cosmed K4b2, Roma, Italy) validated by McLaughlin et al. (2001). The criteria used to determine VO2 max were a plateau in VO2 despite an increase in power output, an RER above 1.1, and a heart rate above $90 \%$ of the predicted maximal heart rate (Howley et al. 1995). Maximal oxygen uptake ( VO2 max) was determined by the average of the last 4 highest VO2 values recorded. Ventilatory thresholds (VT1 and VT2) were determined according to the method described by Wasserman et al. (1973).

\section{Randomization to treatment group}

After the evaluation of VO2 max, 2 groups of 8 elderly people were randomly assigned in a double-blind process to 1 of 2 treatment groups: antioxidant supplementation (As group, $n=8$ ) (Isoxan Senior, NHS, Rungis, France) or placebo (Pl group, $n=8$ ) (for details, see Table 1). Treatments began 21 days before the resistance fatiguing exercise. All subjects were instructed to maintain their normal diets during the 21 days of supplementation. Capsule consumption, assessed by counting the capsules provided on return of pill bottles, was 99.3\% for the Pl group and $99.5 \%$ for the As group. The daily supplementation (3 capsules per day) of Isoxan Senior respected the recommended daily allowances of elderly people. 


\section{Second session: cycling control exercise and 1RM knee extension evaluation}

During their second visit to the laboratory (i.e., after 21 days of supplementation), the subjects underwent a 10-min control cycling exercise test (CTRL) at a selfselected cadence on the cycling ergometer. For this test, a heavy resistance was imposed on the subjects, corresponding to Pexercise $=(P V T 1+P V T 2) / 2$, where $P$ is power output. Such exercise intensity is classically associated with a VO2SC and a muscular activity sufficient to obtain an EMG signal sensitive to experimental conditions (Vercruyssen et al. 2008). During this exercise, VO2 was recorded continuously using the same cycle-to-cycle metabolic portable system as during the first session, and surface EMG was recorded on the muscles of the dominant leg. Immediately before (MVCCTRL) and after (MVCCTRL Post) the CTRL, subjects were placed in a seated position and were strapped securely into the test chair to perform an isometric MVC knee extension and flexion of their dominant leg using an isometric ergometer (Biodex system 3, Biodex Medical, Shirley, N.Y.). Subjects sat with a 908 knee angle (08 as full leg extension), with the ankle attached to the ergometer arm. The knee axis was aligned with the ergometer axis. EMG activity was recorded on the vastus medialis (VM) and vastus lateralis (VL) muscles during the knee extensors MVC and on the biceps femoris (BF) during the knee flexors MVC. Subjects performed 3 MVC of short duration (5 s) of the knee flexors and extensors muscles, separated by a 60-s period of rest. The best performance consecutive to the 3 trials determined the maximal isometric voluntary torque (MVC, in Newtons). One hour after the previous test, subjects were evaluated on their 1 repetition maximum (1RM) during a knee-extension exercise on a horizontal leg press (Technogym, Gambettola, Italy), according to the method described by Bishop et al. (1999). After 10 submaximal warm-up contractions, the resistance was gradually increased until the subject could only achieve full knee extension (1RM) once and not twice. This was recorded as the subject's 1RM.

\section{Third session: fatiguing strength session and cycling exercise}

Twenty-four to $48 \mathrm{~h}$ after the second session, subjects had to perform a fatiguing strength exercise composed of 10 sets of 10 repetitions on the horizontal leg press, at an intensity of 70\% of their 1RM (Ferri et al. 2006). The rest between sets was $90 \mathrm{~s}$. Neutral position was defined as an angle of knee flexion of 1108 measured with a goniometer. The exercise consisted of a 1-s concentric contraction followed by a 3-s eccentric contraction. Each contraction started from a position of 1108 of knee flexion to reach to a knee flexion of 1708. The subjects were encouraged verbally to carry out all the sets. All subjects were able to complete the 10 sets of 10 repetitions. Immediately after the fatiguing exercise, all subjects performed $10 \mathrm{~min}$ of cycling at the same intensity as that of the CTRL. Immediately before the fatiguing exercise (MVCPre Fatigue) and after the cycling exercise (MVCPost Fatigue), subjects performed 3 MVC of short duration (5 s) of the knee extensor muscles. During the cycling exercise, VO2 and the surface EMG of the muscles of the dominant leg were recorded.

\section{Analysis and treatment of recorded data}

\section{Metabolic parameters}

For each subject and each session of cycling, breath-bybreath data were time aligned. Resting data were obtained by averaging the values recorded over the 3-min rest period prior to exercise. Gross efficiency was calculated using the following equation (Gaesser and Brooks 1975): 


$$
\mathrm{GE}=\frac{\mathrm{WR}}{E} \times 100
$$

where GE is gross efficiency (\%), WR is work rate in joules, and $E$ is energy expenditure in joules.

Energy expenditure was estimated from consumption values recorded during the last 2 min of exercise and from the difference in blood lactate ([La]b) values between the beginning and the end of exercise ([DLa]b). For oxygen values, energy expenditure was calculated from tables that provide calorific equivalents of _VO2 based on the RER. The estimate for [DLa]b was based mainly on the assumption of an energetic equivalent for lactate of $62.7 \mathrm{~J} \_\mathrm{kg}-1$ of body mass for a 1 mmol_L-1 increase in [La]b (di Prampero 1986).

The difference in VO2 between minute 10 and minute 3 of each work bout was chosen to estimate the amplitude of the slow component of _VO2 kinetics because exponential modeling using a single trial can be too noisy to describe relatively small changes in kinetics following perturbation (Bearden and Moffatt 2001).

\section{Muscular activity}

Dynamic contractions (cycling sessions)

EMG activity of the VM, VL, and BF muscles of the dominant leg, selected for their high contribution to the propulsive cycling task (Ryan and Gregor 1992), were monitored using bipolar surface electrodes (Blue sensor Q-OO-S, A/S, Oelstykke, Denmark). These pairs of AgAgCl pregelled electrodes (interelectrode distance, $0 \mathrm{~mm}$; area of electrode, $50 \mathrm{~mm} 2$ ) were applied along the fibers over the bellies of the 3 muscles, when each was contracted, according to the recommendations of Rainoldi et al. (2004). A low skin impedance $(<5 \mathrm{kO})$ was obtained by abrading and cleaning it with alcohol wipe. The impedance was measured with a multimeter (Isotech, IDM $93 \mathrm{~N}$, England). The electrodes were secured with surgical tape and cloth wrap to minimize disruption during movement. A ground electrode was placed on a bony site over the patella. To ensure that the electrodes were at precisely the same place for each testing session, we marked the electrode location on the skin with an indelible marker. EMG signals were preamplified close to detection sites (common mode refection ratio, $100 \mathrm{db}$; $Z$ input, $10 \mathrm{GO}$; gain, 600) with a bandwidth frequency ranging from $6 \mathrm{~Hz}$ to $1600 \mathrm{~Hz}$. Prior to acquisition, the signal was filtered with an antialiasing filter (0-500 Hz). EMG data were then collected from each muscle, digitized through an acquisition board (DT 9800-series, Data Translation, Marlboro, Mass., USA) at a 1000-Hz sampling frequency, and stored on a computer to be analyzed using custom-written add-on software (Origin 6.1, OriginLab, Northampton, Mass., USA). Integrated EMG values (iEMG) were collected from each muscle during 40 consecutive crank cycles between minutes 4 and 5, and between minutes 9 and 10 of the 2 trials. Because free-cycling cadence choice could affect burst duration, iEMG values were normalized relative to the burst duration (iEMG/burst duration). These values were named “iEMG flow, QiEMG”' and were considered the measurement of muscle activity (Vercruyssen et al. 2008). Subsequently, we made an average with the values of the first period (minutes 4 to 5) and the second period (minutes 9 to 10) of the 2 cycling exercises.

For each muscle, 4 parameters were analyzed systematically: beginning (Burst Onset) and ending (Burst Offset) times of the burst activity, burst duration, and iEMG flow values (QiEMG). To calculate these parameters, raw data were unbiased, full-wave rectified, and smoothed with a 10$\mathrm{Hz}$ low-pass filter to create a linear envelope. The criteria for the onset and offset values were based on a minimum threshold of 3 SDs from the resting baseline and a minimum burst duration 
of $50 \mathrm{~ms}$. Upon reaching the determined threshold, the muscle was considered active, and the muscle burst duration was defined as the duration between the onset and offset values. Coactivation duration (in \% of agonistic duration) between agonistic (VM and VL) and antagonistic (BF) muscles was also calculated for each muscle pair (BF-VM, BF-VL).

Isometric contractions (MVC sessions)

Median frequency (MF), mean power frequency (MPF), and root mean square (RMS) values were assessed during the isometric MVCs for each extensor muscle (VM and VL). The EMG signals collected within the first and last seconds of each 5-s isometric contraction were not used for analysis because of knee movement that may have occurred at the initiation and completion of the test. Therefore, a 3-s window of EMG signals was used for the power spectrum analysis. A fast Fourier transformation was performed on consecutive time windows of 0.5-s duration over this portion (i.e., middle $3 \mathrm{~s}$ ) of each contraction. MF, MPF, and RMS values were determined from each of the time windows and mean values were then calculated for each muscle over the contraction period.

\section{Statistical analysis}

All data were expressed as means \pm SD. A 2-way analysis of variance (ANOVA) (group session) for repeated measures was performed to analyze the effect of groups and heavy exercise using MVC, muscular activity parameters, cadence, gross efficiency, and VO2SC as dependent variables. A Tukey post-hoc test was used to determine any differences among the pre- and postheavy-fatiguing exercise and groups. For all statistical analyses, $p<0.05$ was accepted as the level of significance.

\section{Results}

\section{Metabolic parameters}

Metabolic parameters recorded during cycling before and after the fatiguing exercise are presented in Table 2. No significant effects of fatigue and supplementation were observed on baseline VO2 (_VO2b). Cycling gross efficiency was reduced significantly after the fatiguing exercise whatever the group, but this decrease was significantly smaller for the As group (As, $5.4 \%$ vs. $\mathrm{Pl},-9.9 \%$ ). Furthermore, VO2SC values were significantly affected by fatigue and supplementation. For both groups,_VO2SC values were higher after fatigue. However, these increases were significantly smaller for the As group when compared with the Pl group ( VO2SC, As $+3.8 \%$ vs. $\mathrm{Pl}+24.4 \%$ ). (Fig. 2).

\section{Muscular activity}

\section{Isometric contractions}

Results indicate no effect of supplementation on MVC, before and after the fatiguing exercise. For both groups, a significant and similar decrease in MVC was noted after fatigue (As, $-10.9 \%$ vs. $\mathrm{Pl},-11.3 \%$ ) (Fig. 3). In both groups, the MVC loss after fatigue was associated with a significant decrease in the EMG frequency parameters (MF and MPF) for VM and VL (Table 3). This shift towards lower frequencies was significantly less important for the As group when compared with the Pl group.

\section{Dynamic contractions}


A significant increase in cycling cadence was observed after the fatiguing exercise, whatever the group (As, $+11.6 \%$ vs. Pl, $+5.6 \%$ ), without any supplementation effect. Changes in iEMG flow and antagonistic coactivations during the 2 high-intensity cycling bouts are presented in Table 4. QiEMG of agonistic muscle (VL) increased after fatigue for all subjects. However, this increase was significantly less important for the As group (As, $+6.6 \%$ vs. Pl, $+12.7 \%$ ). In contrast, QiEMG of antagonistic muscle (BF) decreased in similar proportions for the As and Pl groups (Fig. 4). Furthermore, no significant effects of fatigue or nutritional supplementation were observed on coactivation durations (expressed in percentage of antagonistic duration) between agonist and antagonist muscles.

\section{Discussion}

The purpose of this study was to examine the effects of vitamin and mineral supplementation on muscular activity and cycling efficiency in elderly people, during cycling sessions performed before and after a fatiguing strength exercise. The main findings of the present study indicated an interaction effect between fatigue and supplementation on cycling efficiency during a 10-min constant-intensity cycling task (i.e., above ventilatory threshold) and on muscular activity recorded during dynamic and isometric exercises. During cycling exercise, fatigue induced a decrease in gross efficiency and an increase in VO2SC and agonistic muscular activity for both the As and the Pl groups, but these changes were significantly lower for the As group. Moreover, despite a similar decrease in maximal isometric voluntary torque after fatigue, the decrease in (MF and MPF) was significantly lower for the As group when compared with the Pl group. These results suggest a significant effect of vitamin and mineral supplementation on exercise tolerance in well-trained elderly subjects.

In the literature, the influence of nutritional supplementation has been studied during fatiguing exercises such as long distance activities, where eccentric muscle contractions are dominant. Eccentric contractions involve generating force while lengthening active muscle. Such activities are known to produce severe damages to muscular properties. Indeed, muscle contractions can result in disruption in connective tissue and myocellular components, including the sarcolemma, myofibrils, and cytoskeleton (Gandevia 2001). Exercise- induced damages are classically accompanied by a decrease in MVF. For example, maximal isometric kneeextension MVF has been reported to decrease by $30 \%$ after a 65-km ultramarathon (Millet et al. 2002), by $24 \%$ after a 30-km running race (Millet et al. 2003), and by 36.5\% after a 55-km trail running race (Gauché et al. 2006). Moreover, Lavender and Nosaka (2006) have reported a significant reduction in MVF concomitant with an increase in plasma creatine kinase, especially after an eccentric exercise composed of 6 sets of 5 reps of dumbbell exercise of the elbow flexors eccentrically with 1 arm and concentrically with the other. Similarly, our results show a significant and similar decrease in MVF after the repeated concentric-eccentric contractions on the leg press, for 2 groups (As, $10.9 \%$ vs. $\mathrm{Pl},-11.3 \%)$.

In the present study, the reduction in MVF is smaller than alterations reported in previous studies analyzing fatigue after long-duration exercise; however, the significant reduction in MVC indicates the appearance of fatigue in both populations. Furthermore, the MVF loss after fatigue was associated with a significant decrease in the EMG frequency parameters (MPF and MF) for the VL and VM muscles in both groups. Classically, a compression of EMG power spectrum occurs immediately after a low-force voluntary protocol (De Luca 1984; Linnamo et al. 2000). This phenomenon is commonly explained by a decrease in muscle fiber propagation velocity, 
related to a decrease in blood pH (De Luca 1984; Linnamo et al. 2000), and (or) in motorunit synchronization (De Luca 1984).

In our study, the effect of fatigue was also observed during cycling exercise, with an increase in muscle activity associated with changes in metabolic parameters. Our results indicate a decrease in gross efficiency after the fatiguing exercise, associated with an increase of VO2SC amplitude. On the one hand, the role of gross mechanical efficiency in endurance performance has been classically highlighted. When cycling at 70-80 r_min-1, gross efficiency is positively related to type I muscle fiber recruitment, which has great potential to improve endurance performance (Faria et al. 2005; Hausswirth and Brisswalter 2008). Our results agree with previous research indicating that with fatigue comes a decrease in efficiency of locomotion, associated with an alteration in muscular performance of the locomotors pattern and a decrease in endurance performance (Millet et al. 2000). On the other hand, VO2SC has been described as an important determinant of exercise tolerance (Carter et al. 2000). This parameter indicates that the efficiency with which the body uses oxygen to produce energy is progressively lost while exercise continues at exactly the same speed. Why physiologic mechanisms affect the VO2SC during heavy exercise remains unclear. However, the main factor evoked in the literature is muscle activity that represents 86\% of VO2SC (Poole et al. 1994), with the recruitment of subsidiary muscles or an additional recruitment of muscle fibers (Carter et al. 2000; Vercruyssen et al. 2008). Therefore, for both groups, the increase in EMG agonistic activity (VL) after fatigue could be related to the changes in metabolic parameters, indicating an alteration of muscular and metabolic properties after the fatiguing task, for all subjects. However, one interesting finding is that these fatigueinduced muscular and metabolic alterations were systematically slower for the supplemented group (As), suggesting a beneficial effect of the micronutritional supplementation.

Several factors could be related to an alteration in muscular properties with fatigue and, among those factors, several studies have implicated free radicals (Warren et al. 1992; Duarte et al. 1994; Mastaloudis et al. 2004; Figueiredo et al. 2008). After initial eccentric-related damages, oxidative stress could be increased by the infiltration of neutrophils and macrophages into muscle. As a consequence, oxidative stress is produced in excess and overwhelms antioxidant defenses, resulting in uncontrolled oxidation of the muscle's cellular constituents (Mastaloudis et al. 2004). In our study, for both groups, we observed a significant and similar decrease in MVF after the repeated concentric-eccentric contractions on the leg press. This result agreed with studies on the effect of supplementation on muscle-force decrease after fatigue in young subjects (Warren et al. 1992; Jakeman and Maxwell 1993; Gauche' et al. 2006). In these studies, the antioxidant supplementation did not attenuate the loss of force immediately after a fatiguing concentric-eccentric exercise, but increased muscle recovery capacities in supplemented subjects when compared with a placebo group. For example, recently, in young trained subjects, Gauché et al. (2006) observed a faster recovery of MVF $48 \mathrm{~h}$ after an ultra-trail event when subjects were supplemented with a vitamin and mineral complex. Based on these results, we could suggest in our study a positive effect of the supplementation on muscle performance during the subsequent cycling exercise by limiting muscular damages during the fatiguing exercise. In our study, in both groups, the MVC loss after fatigue was associated with a significant decrease of EMG frequency parameters (MPF and MF) for VL and VM muscles. Moreover, this shift to lower frequencies consecutive to fatigue was systematically lower for the As group than for the Pl group. This result confirms the beneficial effect of supplementation because it limits muscle fatigue after concentric-eccentric exercise. Using dietary manipulation, several studies have indicated that vitamin $\mathrm{E}$ and vitamin $\mathrm{C}$ could have a protective effect against eccentric exercise-induced muscular damages (Maxwell et al. 1993). Within this context, vitamin supplementation is thought 
to act in combination with glutathione to protect cell structure from oxygen radical attack at the surface of the membrane. One interesting result of our study is that this positive effect is also observed on muscular and metabolic parameters recorded during a subsequent cycling exercise, indicating a better exercise tolerance in supplemented subjects.

Among factors affecting exercise capacity with ageing, the role of a progressive accumulation of reactive oxygen species has been widely described in the literature (Ji 2007). This effect could be enhanced in master athletes because regular endurance training sessions lead to repeated expositions to oxidative stress, generated by important VO2 and cellular damages consecutive to muscular contractions (Mastaloudis et al. 2004; Ji 2007). Based on these observations, antioxidant supplementation could be an interesting strategy to enhance antioxidant status and counteract the oxidative stress in master athletes. In the present study, we have tested the influence of vitamin and mineral supplementation on exercise tolerance assessed by gross efficiency or VO2SC and muscular activity during a cycling session performed immediately after a fatiguing concentric-eccentric exercise. Results indicate a decrease in efficiency and an increase in VO2SC associated with an increase of EMG values in both groups. These results are consistent with those of previous studies in which fatigue is classically associated with an increase in VO2 and muscular activity to maintain a given intensity (Hausswirth et al. 2000). However, in our study, metabolic and muscular alterations were systematically lower for the As group, compared with the $\mathrm{Pl}$ group. Moreover, the positive effect of the supplementation on muscle activity was observed only on agonist (VL) and not antagonist muscle (BF). This result could be explained mainly by the high training status of our subjects. A lower antagonistic activity during dynamic exercises is classically observed in trained subjects and indicates a good muscular efficiency consecutive to numerous years of training (Chapman et al. 2008). Therefore, our results give indirect evidence in young subjects of the protective effect of antioxidants present in the vitamin and mineral complex, as previously suggested in the literature (Gauché et al. 2006). This result could be particularly interesting to master athletes because ageing could potentiate the oxidative stress consecutive to eccentric exercise or high VO2 during endurance training.

\section{Conclusion}

Our study aimed to examine the influence of vitamin and mineral supplementation on metabolic parameters and muscular activity during a heavy cycling trial performed by elderly people after a fatiguing concentric-eccentric exercise. One originality of the present study was the recruitment of elderly people regularly trained in endurance (i.e., master athletes). Indeed, studies on elderly people have generally been performed with sedentary elderly people. Within this framework, we have observed a beneficial effect of nutritional supplementation on muscular activity and cycling efficiency of master athletes engaged in heavy exercises, by reducing the fatigue phenomenon and enhancing subsequent exercise tolerance.

\section{Acknowledgements}

This research received financial support from the NHS laboratory located in Rungis (Val-deMarne), France.

\section{References}


Bearden, S.E., and Moffatt, R.J. 2001. VO(2) slow component: to model or not to model? Med. Sci. Sports Exerc. 33(4): 677- 680. PMID:11283448.

Bishop, D., Jenkins, D.G., Mackinnon, L.T., McEniery, M., and Carey, M.F. 1999. The effects of strength training on endurance performance and muscle characteristics. Med. Sci. Sports Exerc. 31(6): 886-891. doi:10.1097/00005768-199906000-00018. PMID:10378917.

Carter, H., Jones, A.M., Barstow, T.J., Burnley, M., Williams, C., and Doust, J.H. 2000. Effect of endurance training on oxygen uptake kinetics during treadmill running. J. Appl. Physiol. 89: 1744-1752. PMID:11053321.

Chapman, A.R., Vicenzino, B., Blanch, P., and Hodges, P.W. 2008. Patterns of leg muscle recruitment vary between novice and highly trained cyclists. J. Electromyogr. Kinesiol. 18(3): 359-371. doi:10.1016/j.jelekin.2005.12.007. PMID:17258470.

Daly, D.J., and Cavanagh, P.R. 1976. Asymmetry in bicycle ergometer pedaling. Med. Sci. Sports Exerc. 8(3): 204-208. doi:10. 1249/00005768-197600830-00013.

De Luca, C.J. 1984. Myoelectrical manifestations of localized muscular fatigue in humans. Crit. Rev. Biomed. Eng. 11(4): 251- 279. PMID:6391814.

Deley, G., Kervio, G., Van Hoecke, J., Verges, B., Grassi, B., and Casillas, J.M. 2001. Effects of a one-year exercise training program in adults over 70 year old: a study with a controm group. Aging Clin. Exp. Res. 19: 310-315. PMID:17726362.

di Prampero, P.E. 1986. The energy cost of human locomotion on land and in water. Int. J. Sports Med. 7(2): 55-72. doi:10.1055/ s-2008-1025736. PMID:3519480.

Duarte, J.A., Carvalho, F., Bastos, M.L., Soares, J.M., and Appell, H.-J. 1994. Do invading leucocytes contribute to the decrease in glutathione concentrations indicating oxidative stress in exercised muscle, or are they important for its recovery? Eur. J. Appl. Physiol. 68(1): 48-53. doi:10.1007/BF00599241.

Faria, E.W., Parker, D.L., and Faria, I.E. 2005. The science of cycling: factors affecting performance - part 2. Sports Med. 35(4): 313-337. doi:10.2165/00007256-200535040-00003. PMID: 15831060.

Ferri, A., Narici, M., Grassi, B., and Pousson, M. 2006. Neuromuscular recovery after a strength training session in elderly people. Eur. J. Appl. Physiol. 97(3): 272-279. doi:10.1007/s00421006- 0168-y. PMID:16572331.

Figueiredo, P.A., Mota, M.P., Appell, H.J., and Duarte, J.A. 2008. The role of mitochondria in aging of skeletal muscle. Biogerontology, 9(2): 67-84. doi:10.1007/s10522-007-9121-7. PMID: 18175203.

Gaesser, G.A., and Brooks, G.A. 1975. Muscular efficiency during steady-rate exercise: effects of speed and work rate. J. Appl. Physiol. 38(6): 1132-1139. PMID:1141128. 
Gandevia, S.C. 2001. Spinal and supraspinal factors in human muscle fatigue. Physiol. Rev. 81(4): 1725-1789. PMID:11581501.

Gauché, E., Lepers, R., Rabita, G., Leveque, J.-M., Bishop, D., Brisswalter, J., and Hausswirth, C. 2006. Vitamin and mineral supplementation and neuromuscular recovery after a running race. Med. Sci. Sports Exerc. 38(12): 2110-2117. doi:10.1249/ 01.mss.0000235351.01438.5a. PMID:17146317.

Goldfarb, A.H., Bloomer, R.J., and McKenzie, M.J. 2005. Combined antioxidant treatment effects on blood oxidative stress after eccentric exercise. Med. Sci. Sports Exerc. 37(2): 234-239. doi:10.1249/01.MSS.0000152887.87785.BE. PMID:15692318.

Hausswirth, C., and Brisswalter, J. 2008. Strategies for improving performance in long duration events: Olympic distance triathlon. Sports Med. 38(11): 881-891. doi:10.2165/00007256200838110- 00001. PMID:18937520.

Hausswirth, C., Brisswalter, J., Vallier, J.M., Smith, D., and Lepers, R. 2000. Evolution of electromyographic signal, running economy, and perceived exertion during different prolonged exercises. Int. J. Sports Med. 21(6): 429-436. doi:10.1055/s-2000- 3832. PMID:10961519.

Howley, E.T., Bassett, D.R., Jr., and Welch, H.G. 1995. Criteria for maximal oxygen uptake: review and commentary. Med. Sci. Sports Exerc. 27(9): 1292-1301. PMID:8531628.

Jakeman, P., and Maxwell, S. 1993. Effect of antioxidant vitamin supplementation on muscle function after eccentric exercise. Eur. J. Appl. Physiol. Occup. Physiol. 67(5): 426-430. doi:10. 1007/BF00376459. PMID:8299614.

Ji, L.L. 2007. Antioxidant signaling in skeletal muscle: a brief review. Exp. Gerontol. 42(7): 582593. doi:10.1016/j.exger.2007. 03.002. PMID:17467943.

Lattier, G., Millet, G.Y., Martin, A., and Martin, V. 2004. Fatigue and recovery after highintensity exercise. Part II: Recovery interventions. Int. J. Sports Med. 25(7): 509-515. doi:10.1055/s- 2004-820946. PMID:15459831.

Lavender, A.P., and Nosaka, K. 2006. Changes in fluctuation of isometric force following eccentric and concentric exercise of the elbow flexors. Eur. J. Appl. Physiol. 96(3): 235-240. doi:10.1007/s00421-005-0069-5. PMID:16249919.

Lepers, R. 2008. Analysis of Hawaii ironman performances in elite triathletes from 1981 to 2007. Med. Sci. Sports Exerc. 40(10): 1828-1834. doi:10.1249/MSS.0b013e31817e91a4. PMID: 18799994.

Lexell, J. 1995. Human aging, muscle mass, and fiber type composition. J. Gerontol. A Biol. Sci. Med. Sci. 50: 1-16. PMID: 7493202. 
Linnamo, V., Bottas, R., and Komi, P.V. 2000. Force and EMG power spectrum during and after eccentric and concentric fatigue. J. Electromyogr. Kinesiol. 10(5): 293-300. doi:10.1016/ S10506411(00)00021-3. PMID:11018439.

Louis, J., Hausswirth, C., Bieuzen, F., and Brisswalter, J. 2009. Muscle strength and metabolism in master athletes. Int. J. Sports Med. 30(10): 754-759. doi:10.1055/s-0029-1231046. PMID: 19685414.

Mastaloudis, A., Morrow, J.D., Hopkins, D.W., Devaraj, S., and Traber, M.G. 2004. Antioxidant supplementation prevents exercise- induced lipid peroxidation, but not inflammation, in ultramarathon runners. Free Radic. Biol. Med. 36(10): 1329-1341. doi:10.1016/j.freeradbiomed.2004.02.069. PMID:15110397.

Maxwell, S.R., Jakeman, P., Thomason, H., Leguen, C., and Thorpe, G.H. 1993. Changes in plasma antioxidant status during eccentric exercise and the effect of vitamin supplementation. Free Radic. Res. Commun. 19: 191-201.

McLaughlin, J.E., King, G.A., Howley, E.T., Bassett, D.R., Jr., and Ainsworth, B.E. 2001. Validation of the COSMED K4 b2 portable metabolic system. Int. J. Sports Med. 22(4): 280 284. doi:10.1055/s-2001-13816. PMID:11414671.

Millet, G., Lepers, R., Lattier, G., Martin, V., Babault, N., and Maffiuletti, N. 2000. Influence of ultra-long-term fatigue on the oxygen cost of two types of locomotion. Eur. J. Appl. Physiol. 83(4-5): 376-380. doi:10.1007/s004210000313. PMID: 11138578.

Millet, G.Y., Lepers, R., Maffiuletti, N.A., Babault, N., Martin, V., and Lattier, G. 2002. Alterations of neuromuscular function after an ultramarathon. J. Appl. Physiol. 92(2): 486-492. PMID: 11796655.

Millet, G.Y., Martin, V., Lattier, G., and Ballay, Y. 2003. Mechanisms contributing to knee extensor strength loss after prolonged running exercise. J. Appl. Physiol. 94(1): 193-198. PMID: 12391039.

Poole, D.C., Barstow, T.J., Gaesser, G.A., Willis, W.T., and Whipp, B.J. 1994. VO2 slow component: physiological and functional significance. Med. Sci. Sports Exerc. 26(11): 13541358. PMID:7837956.

Rainoldi, A., Melchiorri, G., and Caruso, I. 2004. A method for positioning electrodes during surface EMG recordings in lower limb muscles. J. Neurosci. Methods, 134(1): 37-43. doi:10. 1016/j.jneumeth.2003.10.014. PMID:15102501.

Ryan, M.M., and Gregor, R.J. 1992. EMG profiles of lower extremity muscles during cycling at constant workload and cadence. J. Electromyogr. Kinesiol. 2(2): 69-80. doi:10.1016/10506411(92)90018-E.

Sen, C.K., and Hanninen, O. 1994. Physiological antioxidants. In Exercise and Oxygen Toxicity. Edited by C.K. Sen, L. Packer, and O. Hanninen. Elsevier Press, Amsterdam, pp. 89-126. 
Tanaka, H., and Seals, D.R. 2008. Endurance exercise performance in masters athletes: ageassociated changes and underlying physiological mechanisms. J. Physiol. 586(1): 56-63. PMID: 17717011.

Vercruyssen, F., Missenard, O., and Brisswalter, J. 2009. Relationship between oxygen uptake slow component and surface EMG during heavy exercise in humans: Influence of pedal rate. J. Electromyogr. Kinesiol. 19(4): 676-684. PMID:18424174.

Warren, J.A., Jenkins, R.R., Packer, L., Witt, E.H., and Armstrong, R.B. 1992. Elevated muscle vitamin E does not attenuate eccentric exercise-induced muscle injury. J. Appl. Physiol. 72(6): 2168-2175. PMID:1629069.

Wasserman, K., Whipp, B.J., Koyl, S.N., and Beaver, W.L. 1973. Anaerobic threshold and respiratory gas exchange during exercise. J. Appl. Physiol. 35(2): 236-243. PMID:4723033. 


\section{Tables et figures}

Table 1. Component of Isoxan Senior per day and component of placebo.

\begin{tabular}{|c|c|}
\hline \multicolumn{2}{|l|}{ Isoxan Senior $^{a}$} \\
\hline Vitamin C & $106.4 \mathrm{mg}$ \\
\hline Vitamin E & $16.0 \mathrm{mg}$ \\
\hline Vitamin B1 & $1.7 \mathrm{mg}$ \\
\hline Vitamin B2 & $2.0 \mathrm{mg}$ \\
\hline b-carotene & $5.7 \mathrm{mg}$ \\
\hline Vitamin B3 & $20.0 \mathrm{mg}$ \\
\hline Vitamin B6 & $2.6 \mathrm{mg}$ \\
\hline Vitamin B9 & $400 \mu \mathrm{g}$ \\
\hline Vitamin B & $123.2 \mu \mathrm{g}$ \\
\hline Calcium & $133.2 \mu \mathrm{gg}$ \\
\hline Magnesium & $133.2 \mu \mathrm{g}$ \\
\hline Zinc & $16.0 \mathrm{mg}$ \\
\hline Iron & $2 \mathrm{mg}$ \\
\hline Manganese & $4.6 \mathrm{mg}$ \\
\hline Copper & $2.64 \mathrm{mg}$ \\
\hline Selenium & $93.2 \mathrm{\mu g}$ \\
\hline \multicolumn{2}{|l|}{ Placebo } \\
\hline Microcrystalline cellulose & $470 \mathrm{mg}$ \\
\hline Lactose flast & $470 \mathrm{mg}$ \\
\hline Reticuled croscamellose $\mathrm{Na}$ & $30 \mathrm{mg}$ \\
\hline Magnesium stearate & $10 \mathrm{mg}$ \\
\hline Talc & $20 \mathrm{mg}$ \\
\hline Sepifilm LP014 & $1433 \mathrm{mg}$ \\
\hline 3342 Sesispere dry & $22 \mathrm{mg}$ \\
\hline \multicolumn{2}{|c|}{$\begin{array}{l}\text { aOther Isoxan components: microcrystalline cellulose, lactose, croscarmellose, } \\
\text { talc, magnesium stearate, polyvinylpyrrolidone, maltodextrine. Agents of } \\
\text { coating: E464, E570. Dyes: E101, E110, E171. }\end{array}$} \\
\hline
\end{tabular}


Table 2. Metabolic parameters recorded during 2 cycling sessions performed before (pre) and after (post) the fatiguing concentric-eccentric exercise, for antioxidant (As) and placebo (Pl) supplemented groups.

\begin{tabular}{|c|c|c|c|c|}
\hline \multirow[b]{2}{*}{ Metabolic activity } & \multicolumn{2}{|l|}{$\underline{\text { Pre }}$} & \multicolumn{2}{|l|}{ Post } \\
\hline & As & P1 & As & Pl \\
\hline $\bar{V} \mathrm{O}_{2} \mathrm{~b}\left(\mathrm{~mL} \cdot \mathrm{min}^{-1}\right)$ & $321.9 \pm 38.7$ & $349.9 \pm 84.5$ & $316.1 \pm 30.1$ & $308.7 \pm 42.6$ \\
\hline Gross efficiency $(\%)$ & $19.48 \pm 3.4$ & $19.36 \pm 2.1$ & $18.5 \pm 3.3^{*, \dagger}$ & $17.6 \pm 2.3^{*}$ \\
\hline$\dot{V} \mathrm{O}_{2} \mathrm{SC}\left(\mathrm{mL} \cdot \mathrm{min}^{-1}\right)$ & $187.9 \pm 8.1$ & $181.9 \pm 7.4$ & $195 \pm 4.8^{*, \dagger}$ & $225.8 \pm 33.8^{*}$ \\
\hline
\end{tabular}

Note: Values are means \pm SD. VO2b, baseline oxygen uptake; VO2SC, oxygen uptake slow component.

*Significantly different from the prefatigue condition $(p<0.05)$.

\{Significantly different between groups after fatigue $(p<0.05)$.

Table 3. Muscular activity of vastus medialis (VM) and vastus lateralis (VL) muscles, during isometric maximal voluntary contraction (MVC) performed before (Pre) and after (Post) the fatiguing exercise, for the 2 treatment groups (As and $\mathrm{Pl}$ ).

\begin{tabular}{|c|c|c|c|c|}
\hline \multirow[b]{2}{*}{$\begin{array}{l}\text { MVC muscle } \\
\text { activity }\end{array}$} & \multicolumn{2}{|l|}{ Pre } & \multicolumn{2}{|l|}{ Post } \\
\hline & As & $\mathrm{Pl}$ & As & P1 \\
\hline \multicolumn{5}{|l|}{ MPF (Hz) } \\
\hline VM & $127 \pm 83.5$ & $133.7 \pm 69.6$ & $75.6 \pm 9.8^{*, \dagger}$ & $70.3 \pm 18.3^{*}$ \\
\hline VL & $144 \pm 79.8$ & $167.4 \pm 76.1$ & $109 \pm 64.7^{*, \dagger}$ & $72.4 \pm 12.8^{*}$ \\
\hline \multicolumn{5}{|l|}{ MF (Hz) } \\
\hline $\mathrm{VM}$ & $73.2 \pm 12$ & $62.6 \pm 9.1$ & $60.4 \pm 9^{*, \dagger}$ & $53.3 \pm 7.8^{*}$ \\
\hline VL & $69.6 \pm 9.1$ & $72.3 \pm 17.8$ & $60.7 \pm 9.4^{*}$ & $60.7 \pm 15.9^{*}$ \\
\hline \multicolumn{5}{|l|}{ RMS $(\mu \mathbf{V})$} \\
\hline VM & $0.14 \pm 0.047$ & $0.16 \pm 0.04$ & $0.12 \pm 0.022$ & $0.15 \pm 0.05$ \\
\hline VL & $0.18 \pm 0.09$ & $0.11 \pm 0.017$ & $0.17 \pm 0.063^{\dagger}$ & $0.11 \pm 0.03$ \\
\hline
\end{tabular}

Note: Values are means \pm SD. MPF, mean power frequency; MF, median frequency; RMS, root mean square.

*Significantly different from the prefatigue condition $(p<0.05)$.

\{Significantly different between groups after fatigue $(p<0.05)$. 
Table 4. Mechanical parameters and muscular activity of vastus medialis (VM), vastus lateralis (VL), and biceps femoris (BF) during the 2 cycling trials performed before (Pre) and after (Post) fatigue, for the 2 treatment groups (As and $\mathrm{Pl}$ ).

\begin{tabular}{|c|c|c|c|c|}
\hline \multirow[b]{2}{*}{$\begin{array}{l}\text { Cycling muscle } \\
\text { activity }\end{array}$} & \multicolumn{2}{|l|}{$\underline{\text { Pre }}$} & \multicolumn{2}{|l|}{ Post } \\
\hline & As & Pl & As & Pl \\
\hline Cadence $\left(\mathrm{r} \cdot \mathrm{min}^{-1}\right)$ & $81.1 \pm 11.6$ & $79.4 \pm 11.4$ & $90.5 \pm 13.3^{*}$ & $83.9 \pm 11.3^{*}$ \\
\hline Power output (W) & $230.4 \pm 47.8$ & $230.4 \pm 47.8$ & $230.4 \pm 47.8$ & $230.4 \pm 47.8$ \\
\hline \multicolumn{5}{|l|}{ QiEMG (AU) } \\
\hline VM & $112.6 \pm 50$ & $140.2 \pm 50.3$ & $115.7 \pm 53.7$ & $146.6 \pm 59$ \\
\hline VL & $113.7 \pm 47.8$ & $111.2 \pm 52.5$ & $122.4 \pm 59.8^{*}, \dagger$ & $122.5 \pm 50.6^{*}$ \\
\hline $\mathrm{BF}$ & $97.5 \pm 23.1$ & $129.8 \pm 97.3$ & $84.5 \pm 27.3^{*}$ & $106.9 \pm 78.8^{*}$ \\
\hline \multicolumn{5}{|l|}{ Coactivation (\%) } \\
\hline $\mathrm{BF}-\mathrm{VM}$ & $69.5 \pm 23.1$ & $55.2 \pm 25.2$ & $70.6 \pm 22.4$ & $57.4 \pm 25$ \\
\hline $\mathrm{BF}-\mathrm{VL}$ & $78.7 \pm 17$ & $61.5 \pm 28.8$ & $81.8 \pm 9.7$ & $64.6 \pm 27.1$ \\
\hline
\end{tabular}

Note: Values are means \pm SD. Cadence, pedaling cadence (r_min-1); QiEMG, iEMG flow; Coactivation, antagonist muscle coactivation (in \% of agonist duration).

*Significantly different from the prefatigue condition $(p<0.05)$.

\{Significantly different between groups after fatigue $(p<0.05)$.

\section{Figure 1}

Fig. 1. Experimental protocol representation. EMG, electromyographic recording; MVC, maximal voluntary contraction; ctrl, cycling control session; 1RM, 1 repetition maximum; $R$, rest; _VO2 max, maximal oxygen uptake test; As, antioxidant supplemented group; Pl, placebo group.

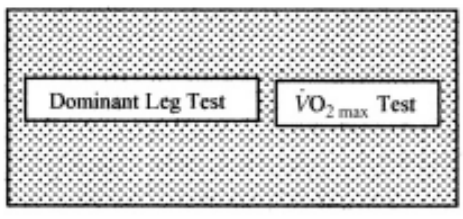

$1^{\text {t }}$ session

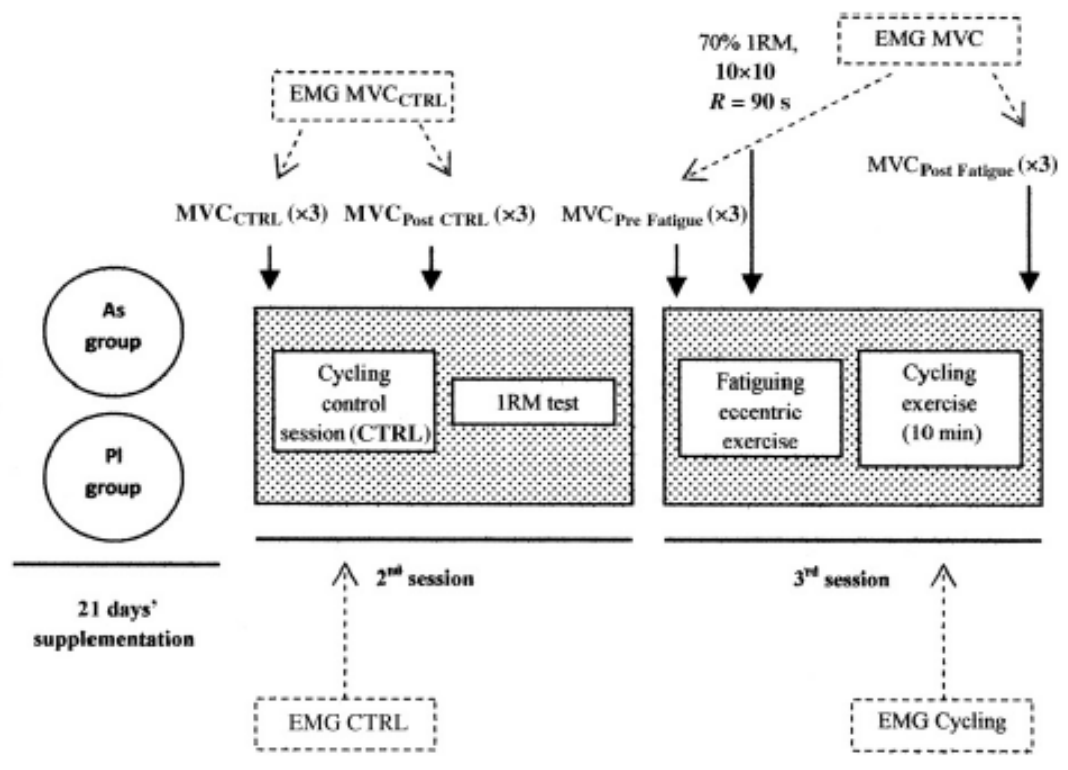




\section{Figure 2}

Fig. 2. Changes in gross efficiency (GE) and oxygen uptake slow component (_VO2SC) during cycling between pre- and postfatigue conditions, in antioxidant (As) and placebo (Pl) supplemented groups. Values are means $\pm \mathrm{SD}$. ${ }^{*}, p<0.05$, significantly different from prefatigue condition; $\{, p<0.05$, significant difference between As and Pl groups.

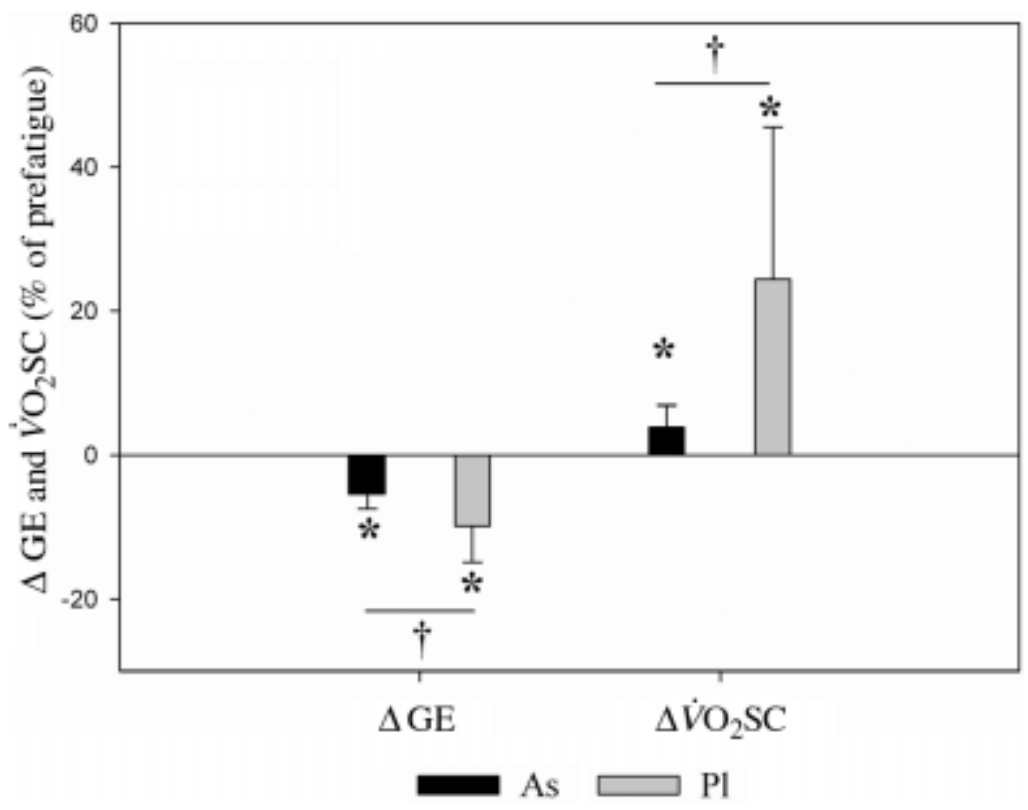

\section{Figure 3}

Fig. 3. Maximal voluntary contraction (MVC) values before (pre) and after (post) the fatiguing exercise, in antioxidant (As) and placebo (Pl) supplemented groups. Values are means $\pm \mathrm{SD} .{ }^{*}, p$ $<0.05$, significantly different from the prefatigue condition.

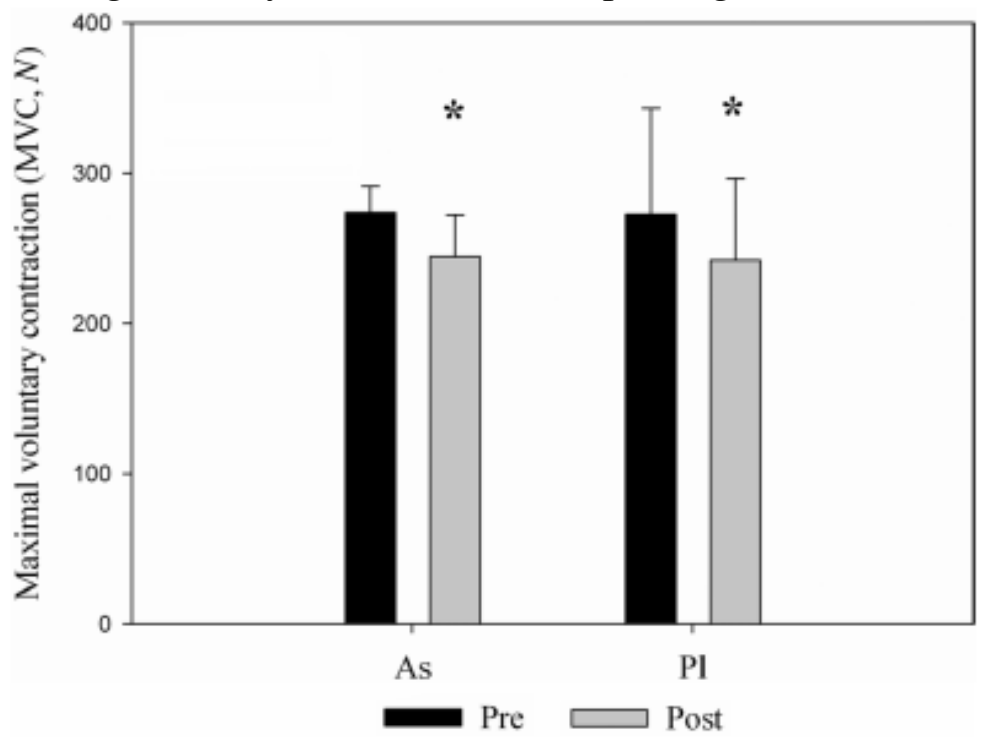




\section{Figure 4}

Fig. 4. Changes in integrated electromyographic signal expressed with regard to burst duration (QiEMG) during cycling between pre and postfatigue conditions, in antioxidant (As) and placebo (Pl) supplemented groups. Values are means $\pm \mathrm{SD} .{ }^{*}, p<0.05$, significantly different from the prefatigue condition; $\{, p<0.05$, significant difference between As and Pl groups. VM, vastus medialis; VL, vastus lateralis; BF, biceps femoris.

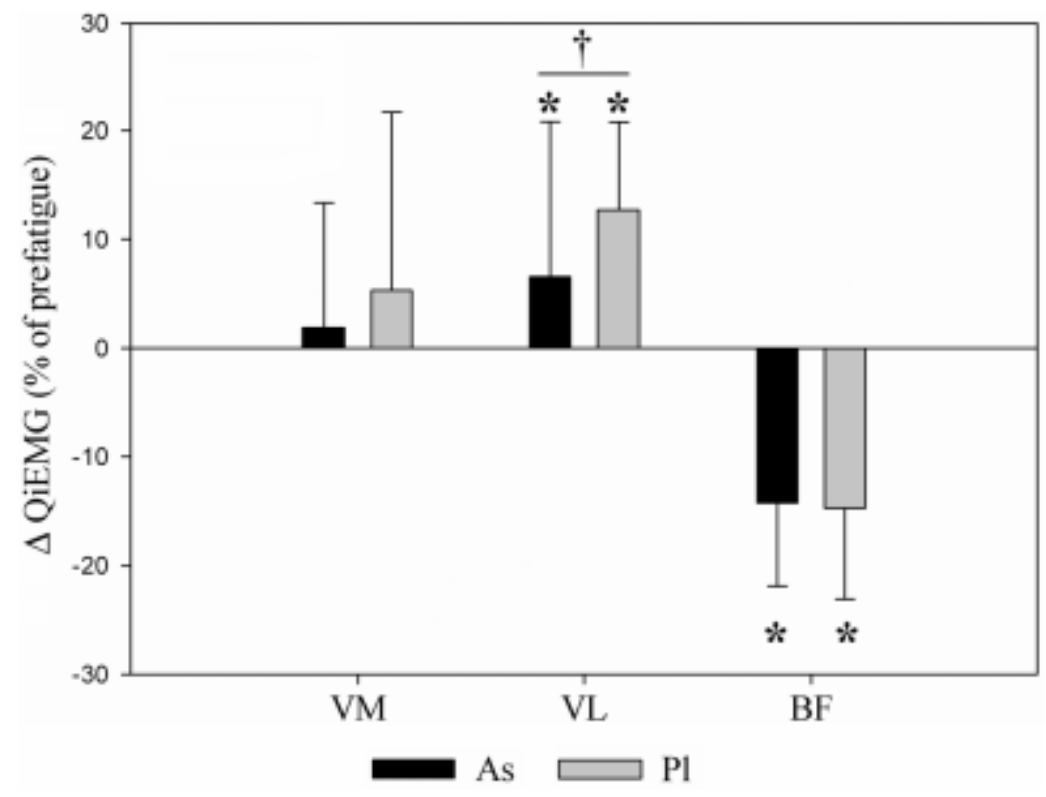

